\title{
Midinfrared photoluminescence and compositional modulation in pentanary GalnAsPSb alloys grown by liquid phase epitaxy
}

\author{
A. Krier, ${ }^{\text {a) }}$ V. M. Smirnov, P. J. Batty, and M. Yin \\ Physics Department, Lancaster University, Lancaster LA1 4YB, United Kingdom \\ K. T. Lai, S. Rybchenko, and S. K. Haywood \\ Department of Engineering, University of Hull, Hull HU6 7RX, United Kingdom \\ V. I. Vasil'ev, G. S. Gagis, and V. I. Kuchinskii \\ Ioffe Physico-Technical Institute, RAS, Politechnicheskaya, 26, St. Petersburg 194021, Russia
}

(Received 10 June 2007; accepted 16 July 2007; published online 20 August 2007)

\begin{abstract}
Room temperature photoluminescence is reported from GaInAsSbP pentanary alloys grown by liquid phase epitaxy on GaSb. The epitaxial layers exhibited emission in the midinfrared between 3 and $4 \mu \mathrm{m}$. Investigation of the structural and photoluminescence properties revealed localization effects associated with potential fluctuations in the pentanary alloy arising from compositional modulation. (C) 2007 American Institute of Physics. [DOI: 10.1063/1.2768892]
\end{abstract}

Continuing interest in the development of III-V materials for use in midinfrared $(2-5 \mu \mathrm{m})$ light sources and detectors is driven by the extensive range of potential applications in this spectral region, including environmental gas monitoring, noninvasive medical diagnosis, tunable IR spectroscopy, and free space optical communications. ${ }^{1}$ The pentanary alloys, such as GaInAsSbP, offer some useful advantages to the device engineer by making available an additional degree of freedom. ${ }^{2,3}$ For a given value of the band gap or lattice constant, properties, such as the refractive index, spin-orbit valence band splitting, band offsets, etc., can be independently varied. This could enable control of optical confinement, carrier leakage, intervalence band absorption, and Auger recombination. However, there have been very few reports on the growth and the luminescence properties of GaInAsSbP pentanary alloys. ${ }^{3,4}$ Recently, however, AlGaInAsSb alloys have been grown by molecular beam epitaxy and effectively used as the barrier layers in room temperature midinfrared quantum well lasers. ${ }^{5}$ Here we report on the liquid phase epitaxial growth of GaInAsSbP pentanary alloys and demonstrate room temperature photoluminescence (PL) in the 3-4 $\mu \mathrm{m}$ spectral range.

Epitaxial growth of pentanary GaInAsPSb single epilayers was carried out from antimony-rich melts onto Ge doped p-type GaSb (100) substrates. Growth melts were prepared from $6 N \mathrm{Sb}$ and $7 N$ In pure metals, while the sources of Ga, As, and $\mathrm{P}$ were undoped polycrystalline $\mathrm{GaSb}$, InAs, and InP binary compounds. The use of antimony as the solvent for liquid phase epitaxy (LPE) growth of GaSb-related alloys has some advantages because it avoids substrate erosion and reduces the formation of $\mathrm{Sb}$ vacancies. ${ }^{6-8}$ Growth was implemented from supercooled melts at temperatures within the interval of 585-600 ${ }^{\circ} \mathrm{C}$. In each case before the deposition of the GaInAsPSb pentanary alloy an undoped GaSb $p$-type buffer layer was grown on the substrate. Structural characterization of the resulting epitaxial layers was done using phase contrast Nomarski microscopy, field emission electron microscopy, and high resolution x-ray diffraction. Subsequent optical investigation of the GaInAsSbP structures in-

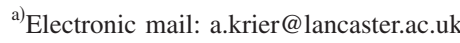

cluded PL spectroscopy using an $\mathrm{Ar}^{+}$ion laser (514 nm) which produced an excitation power density of $20 \mathrm{~W} \mathrm{~cm}^{-2}$ on the sample. Temperature dependent PL measurements over the range of 4-300 K were carried out using conventional lock-in techniques. Additional measurements were made with a Bruker IFS 66/S Fourier transform infrared spectrometer and a $77 \mathrm{~K}$ InSb photodiode detector using a step-scan and a lock-in technique to achieve better resolution at high temperatures.

Using a supercooling of $\Delta T=8-15^{\circ} \mathrm{C}$, the thickness of the resulting epilayers was found to be in the range of 1.0-3.5 $\mu \mathrm{m}$. The GaInAsPSb epilayer surface morphology was flat and mirrorlike when examined using Nomarski microscopy. High-resolution double crystal x-ray diffraction revealed that the lattice mismatch $\Delta a / a=(1.9-4.1) \times 10^{-4}$ at room temperature. The composition of the $\mathrm{Ga}_{1-x} \mathrm{In}_{x} \mathrm{As}_{y} \mathrm{P}_{z} \mathrm{Sb}_{1-y-z}$ epitaxial layers was measured using energy dispersive $\mathrm{X}$-ray microanalysis (EDAX). The alloy compositions obtained are given in Table I together with the corresponding spectral information. The compositional uniformity in the growth direction was found to be approximately constant within the limitations of the EDAX technique $(\sim 1 \%)$.

The PL emission spectra obtained at room temperature from single undoped pentanary epitaxial layers with different alloy compositions is shown in Fig. 1. A broad emission spectrum with a single peak was observed in all cases. (Note that the feature near $4.3 \mu \mathrm{m}$ arises from atmospheric absorption of $\mathrm{CO}_{2}$ in the optical path.) The full width at half maxi-

TABLE I. Alloy compositions (from EDAX) and PL data measured from pentanary alloys grown by LPE.

\begin{tabular}{|c|c|c|c|c|}
\hline \multirow[b]{2}{*}{ Sample } & \multicolumn{2}{|c|}{$\begin{array}{c}\text { PL peak } \\
\text { wavelength }\end{array}$} & \multirow{2}{*}{$\begin{array}{c}\text { Peak width } \\
(\text { FWHM }) \\
(300 \mathrm{~K})(\mathrm{meV})\end{array}$} & \multirow[b]{2}{*}{ Alloy composition } \\
\hline & $300 \mathrm{~K}$ & $77 \mathrm{~K}$ & & \\
\hline S-886 & 4.05 & 3.58 & 57 & $\mathrm{Ga}_{0.03} \mathrm{In}_{0.97} \mathrm{As}_{0.81} \mathrm{P}_{0.06} \mathrm{Sb}_{0.13}$ \\
\hline S-922 & 4.10 & 3.66 & 51 & $\mathrm{Ga}_{0.04} \mathrm{In}_{0.96} \mathrm{As}_{0.83} \mathrm{P}_{0.04} \mathrm{Sb}_{0.13}$ \\
\hline S-923 & 4.05 & 3.66 & 57 & $\mathrm{Ga}_{0.07} \mathrm{In}_{0.93} \mathrm{As}_{0.82} \mathrm{P}_{0.03} \mathrm{Sb}_{0.15}$ \\
\hline S-947 & 3.38 & 3.06 & 61 & $\mathrm{Ga}_{0.03} \mathrm{In}_{0.97} \mathrm{As}_{0.64} \mathrm{P}_{0.17} \mathrm{Sb}_{0.19}$ \\
\hline
\end{tabular}




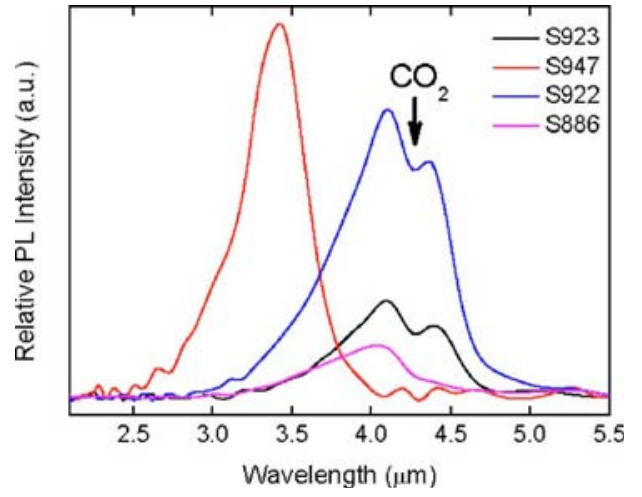

FIG. 1. (Color online) PL emission spectra obtained from $\mathrm{Ga}_{1-x} \mathrm{In}_{x} \mathrm{As}_{y} \mathrm{P}_{z} \mathrm{Sb}_{1-y-z}$ epitaxial layers at $300 \mathrm{~K}$.

mum (FWHM) is $(2-3) k T$ at $300 \mathrm{~K}$. Such PL broadening is associated with alloy disorder and is often used as a semiquantitative measure of the degree of phase separation. ${ }^{9}$ The temperature dependence of the PL emission spectrum from one of the layers (S-886) is shown in Fig. 2. A small blueshift is observed at low temperatures before the peak moves to longer wavelengths. The resulting temperature dependence of the principal peak is shown in Fig. 3. The solid line shows the resulting fit using the Varshni equation, ${ }^{6}$

$$
E_{g}(T)=E_{g}(0)-\alpha T^{2} /(T+\beta),
$$

where $E_{g}(0)$ is the energy gap at $0 \mathrm{~K}$, and $\alpha$ and $\beta$ are the corresponding thermal coefficients. The resulting curve is characteristic of recombination through localized states and has been previously observed in $\mathrm{AlGaAsSb}$ alloys and AlAs/GaAs disordered superlattices. ${ }^{7,10}$ Extrapolation of Eq. (1) to low temperature gives a value of $5 \mathrm{meV}$ for the localization energy. Alloys with different compositions exhibit correspondingly different behaviors with temperature consistent with the recombination of carriers from localized states at different energies. Introducing more $\mathrm{P}$ into the alloy produces additional disorder and increases both the spectral peak width and localization energy (20 meV in S-947 compared with $5 \mathrm{meV}$ in S-923). We do not consider interface recombination to be occurring since we did not observe distinct transitions in the spectra at higher energy as reported for GaInAsSb/GaSb (Ref. 11) and the penetration depth of our $\mathrm{Ar}^{+}$ion laser is easily less than the epitaxial layer thickness.
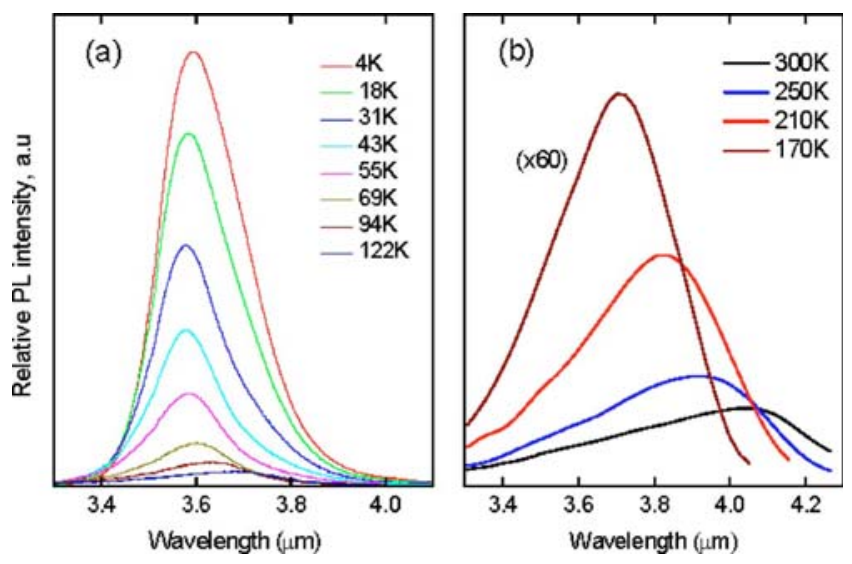

FIG. 2. (Color online) Temperature dependent PL spectra of one of the

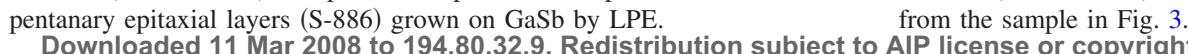

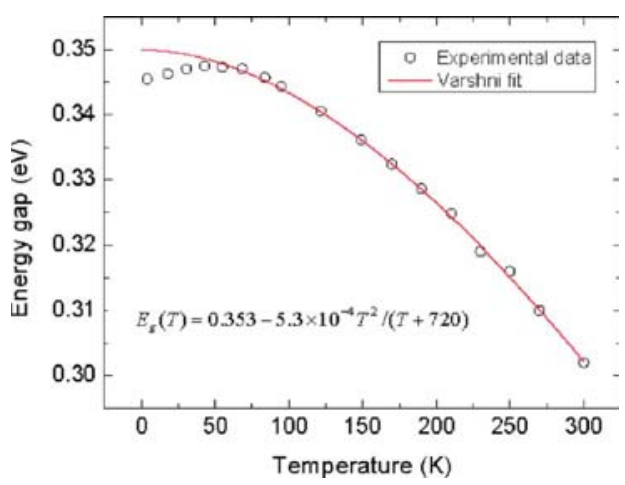

FIG. 3. (Color online) Alloy energy gap variation with temperature for S-886 $\left(\mathrm{Ga}_{0.03} \mathrm{In}_{0.97} \mathrm{As}_{0.81} \mathrm{P}_{0.06} \mathrm{Sb}_{0.13}\right)$.

The temperature dependence of the integrated PL emission intensity in the range of $4-300 \mathrm{~K}$ is shown in Fig. 4. The data cannot be properly fitted by an Arrhenius plot. Instead, the temperature dependence is similar to the behavior observed in disordered crystalline or amorphous semiconductors, ${ }^{12}$

$$
I_{\mathrm{PL}}=I_{0} /\left[1+A \exp \left(T / T_{0}\right)\right]
$$

where $I_{\mathrm{PL}}$ is the normalized peak intensity, $T_{0}$ is the characteristic temperature associated with the ionization energy of localized states, $A$ is a tunneling factor, and $I_{0}$ is the PL intensity in the low temperature limit. Fitting using Eq. (2) yields $T_{0}=32 \mathrm{~K}$ at low temperature. This indicates that the localization energy is approximately $3 \mathrm{meV}$. For temperatures higher than $100 \mathrm{~K}$, carriers are thermally activated and become transferred to occupy other regions with deeper potential and stronger localization. Consequently, the characteristic temperature increases $\left(T_{0}=102 \mathrm{~K}\right)$ corresponding to recombination from carriers localized in states $9 \mathrm{meV}$ below the mobility edge.

Both the temperature dependence of the PL peak wavelength and emission intensity are consistent with recombination through localized band tail states arising from electrostatic potential fluctuations due to compositional inhomogeneities in these alloys. ${ }^{10,13}$ This is not surprising since the alloys produced are close to the boundary of the miscibility gap in this system. Our findings are similar to those for quaternary AlGaAsSb lattice matched to InP, where compositional modulation $(\sim 15 \mathrm{~nm})$ could only be observed using high resolution transmission electron microscopy. ${ }^{13}$ Tight binding calculations of the band structure for pentanary alloys have shown that the addition of $\mathrm{Ga}$ or $\mathrm{P}$ to the alloy

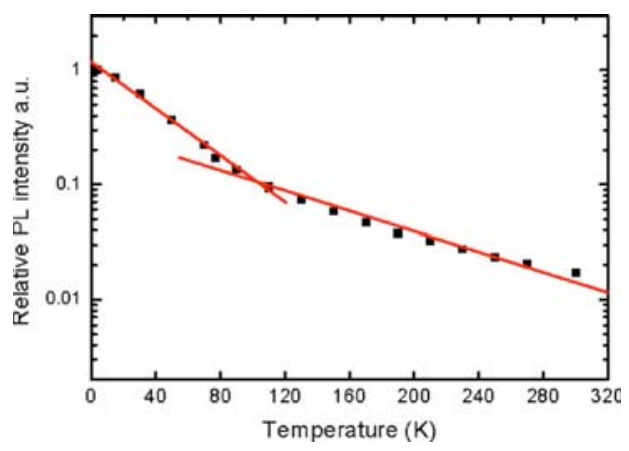

FIG. 4. (Color online) Temperature dependence of PL intensity measured from the sample in Fig. 3. 
widens the band gap and reduces the extent of the conduction band density of states. Whereas, addition of Sb shifts the conduction band to lower energy without changing the density of states. ${ }^{8}$ We suppose therefore that most of the localization effects observed in the PL could be attributed mainly to local variations in the $\mathrm{Sb} / \mathrm{P}$ ratio. In spite of localization effects these alloys exhibit efficient photoluminescence at room temperature covering the $3-4 \mu \mathrm{m}$ wavelength range making them suitable for use as the basis of sources and detectors for midinfrared optoelectronics. Recently, we obtained room temperature electroluminescence near $4 \mu \mathrm{m}$ from $p-i-n$ LEDs fabricated from these pentanary alloys. ${ }^{14}$

In summary, we have demonstrated the LPE growth of pentanary alloys lattice matched onto GaSb substrates. The epitaxial layers exhibit bright photoluminescence in the midinfrared region arising from transitions between localized states within band tails. The temperature dependence of the band gap and thermal quenching behavior is consistent with compositional modulation in these alloys, which are grown near the miscibility gap.

The authors wish to thank EPSRC for financial support (Grant No. GR/S75826/01) and for providing the award of a studentship for one of the authors (P.J.B.).
${ }^{1}$ Mid-Infrared Semiconductor Optoelectronics, edited by A. Krier (Springer, London, 2006), part IV (Applications), pp. 595-730.

${ }^{2}$ V. A. Mishournyi, F. de Anda, A. Y. Gorbachev, and V. I. Vasil'ev, J. Electron. Mater. 28, 959 (1999).

${ }^{3}$ V. I. Vasil"ev, I. P. Nikitina, V. M. Smirnov, and D. N. Tretyakov, Mater. Sci. Eng., B B66, 67 (1999).

${ }^{4}$ M. Aidaraliev, G. G. Zegrya, N. V. Zotova, S. A. Karandashev, B. A. Matveev, N. M. Stus, and G. N. Talalakin, Semiconductors 26, 138 (1992).

${ }^{5}$ M. Grau, C. Lin, O. Dier, C. Lauer, and M.-C. Amann, Appl. Phys. Lett. 87, 241104 (2005).

${ }^{6}$ P. Varshni, Physica (Amsterdam) 34, 149 (1967).

${ }^{7}$ I. T. Yamamoto, K. Sakai, and S. Akiba, IEEE J. Quantum Electron. 15, 684 (1979).

${ }^{8}$ K. Shim, and H. Rabitz, J. Appl. Phys. 85, 7705 (1999).

${ }^{9}$ C. A. Wang, Appl. Phys. Lett. 76 (2000).

${ }^{10}$ D. O. Toginho Filho, I. F. L. Dias, E. Laureto, J. L. Duarte, S. A. Laurenco, L. C. Pocas, S. S. Prabhu, and J. Klem, J. Appl. Phys. 97, 123702 (2005).

${ }^{11}$ K. D. Moiseev and A. Krier, J. Appl. Phys. 90, 3988 (2001).

${ }^{12}$ T. Yamamoto, M. Kasu, S. Noda, and A. Sasaki, J. Appl. Phys. 68, 5318 (1990).

${ }^{13}$ T. H. Chiu, W. T. Tsang, S. N. G. Chu, J. Shah, and J. A. Ditzenberger, Appl. Phys. Lett. 46, 408 (1985).

${ }^{14}$ A. Krier, V. M. Smirnov, P. J. Batty, V. I. Vasil'ev, G. S. Gagis, and V. I. Kuchinskii, Appl. Phys. Lett. 90, 1115 (2007). 